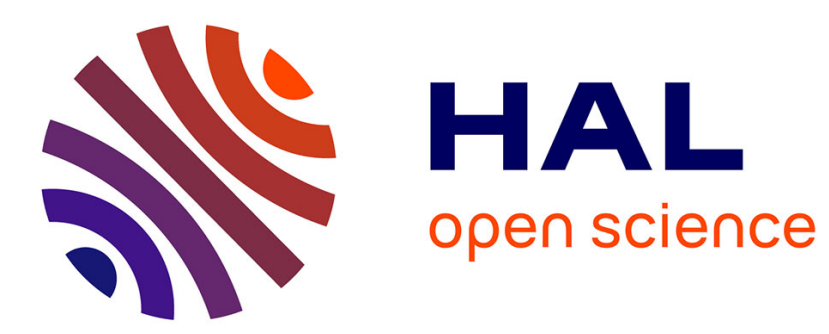

\title{
Upper-limb robotic exoskeletons for neurorehabilitation: a review on control strategies
}

Tommaso Proietti, Vincent Crocher, Agnes Roby-Brami, Nathanael Jarrasse

\section{To cite this version:}

Tommaso Proietti, Vincent Crocher, Agnes Roby-Brami, Nathanael Jarrasse. Upper-limb robotic exoskeletons for neurorehabilitation: a review on control strategies. IEEE Reviews in Biomedical Engineering, 2016, PP (99), pp.1. 10.1109/RBME.2016.2552201 . hal-01302398

\section{HAL Id: hal-01302398 https://hal.sorbonne-universite.fr/hal-01302398}

Submitted on 14 Apr 2016

HAL is a multi-disciplinary open access archive for the deposit and dissemination of scientific research documents, whether they are published or not. The documents may come from teaching and research institutions in France or abroad, or from public or private research centers.
L'archive ouverte pluridisciplinaire HAL, est destinée au dépôt et à la diffusion de documents scientifiques de niveau recherche, publiés ou non, émanant des établissements d'enseignement et de recherche français ou étrangers, des laboratoires publics ou privés. 


\title{
Upper-limb robotic exoskeletons for neurorehabilitation: a review on control strategies
}

\author{
Tommaso Proietti, Vincent Crocher, Agnès Roby-Brami, and Nathanaël Jarrassé
}

\begin{abstract}
Since the late 90s, there has been a burst of research on robotic devices for post-stroke rehabilitation. Robot-mediated therapy produced improvements on recovery of motor capacity; however, so far, the use of robots has not shown qualitative benefit over classical therapist-led training session, performed on the same quantity of movements. Multi degrees of freedom robots, like the modern upper-limb exoskeletons, enables a distributed interaction on the whole assisted limb and can exploit a large amount of sensory feedback data, potentially providing new capabilities within standard rehabilitation sessions.

Surprisingly, most publications in the field of exoskeletons focused only on mechatronic design of the devices while little details were given to the control aspects. On the contrary, we do believe a paramount aspect for robots potentiality lays on the control side. Therefore the aim of this paper is to provide a taxonomy of currently available control strategies for exoskeletons for neurorehabilitation, in order to formulate appropriate questions towards the development of innovative and improved control strategies.
\end{abstract}

Index Terms-Upper-limb robotic exoskeletons, rehabilitation, post-stroke robotic therapy, control strategies.

\section{INTRODUCTION}

Since the end of the nineties, there has been a burst of research on and development of robotic devices for rehabilitation, particularly for the neurorehabilitation of post-stroke patients. Stroke is indeed the second leading cause of death in the world and the leading cause for acquired disability in adults [1], [2]. Stroke survivors are usually left with disabilities, mainly motor impairments on upper-limb movements and loss of hand dexterity, both partially recoverable by undergoing rehabilitation [3]. Rehabilitation has been proven to be effective when it is both intense and involving for the patient [4], and robotics is one possible solution to provide intensity, by increasing the number of repetitions which a therapist could impose, as well as motivation thanks to technology appeal, virtual reality, and gaming [5], [6].

The first robots used for upper-limb rehabilitation, also referred as manipulanda, were only able to guide the motion of patient's hand in the plane (for example the MIT-Manus and MIME platforms [7]). Extensive clinical testing on the InMotion ARM $\odot$ robot (the commercialized version of the MITManus) confirmed the improvements of the motor capacity of the impaired arm after robotic therapy [8]. However, so far, the use of robots did not show extra qualitative benefits (i.e.

T. Proietti, A. Roby-Brami, and N. Jarrassé are with Sorbonne Universités, UPMC Univ. Paris 06, CNRS, UMR 7222, INSERM, the Institute of Intelligent Systems and Robotics (ISIR), 4 place Jussieu, 75005, Paris, France

V. Crocher is with Department of Electrical and Electronic Engineering, University of Melbourne, Australia. improved functional recovery), over conventional therapist-led training session, performing the same quantity of motions [9], [10].

The willingness to address 3D movements and to work at the joint level encouraged the researchers to design robotic exoskeletons for neurorehabilitation. These multiple degrees of freedom (DOF) structures, allowing distributed physical interaction with the whole limb, could potentially provide new capabilities within standard rehabilitation sessions. $\mathrm{Nu}-$ merous upper-limb exoskeletons have therefore been recently developed (around the mid-2000s) but their effects have still been little studied up to now, especially clinically. Indeed, the first and only commercially available upper-limb exoskeleton for rehabilitation (the ArmeoPower@ by Hocoma, based on ARMinIII robot) was only released at the end of 2011 [11]. The two major features of the robotic exoskeletons are their abilities to apply forces distributed along the assisted limbs and to provide reliable joint measurements [10]. Although the physical interaction through multiple interaction points raises interest and fundamental questions from the control point of view, most of the existing control approaches have been developed by only considering the end-point interactions.

While many reviews on robotic exoskeletons are available, most of them are focused on their mechanical features (for example [12], [13], [14]). We do believe that the key characteristic of exoskeletons addressing neurorehabilitation stands in their control strategies which, on top of intrinsic mechanical behaviour of the devices (inertia, friction, backdriveability, etc.), dictate the human-robot interactions. Reviews on highlevel control strategies for neurorehabilitation robots, including both manipulanda and exoskeletons, exist ([15] and [16]), but since these were not targeting the specificities of exoskeletons, many devices and control approaches are missing.

The aim of this paper is therefore to disclose the real interaction capabilities of upper-limb robotic exoskeletons for neurorehabilitation, i.e. their control laws and physical interaction with humans, in order to give researchers in the field an overview of existing works, their possibilities and limitations along with possible orientations for future developments.

\section{Review methodology}

Our review, which rises searching in the main scientific databases (in particular PubMed, ClinicalTrials, IEEE Xplore Digital Library, Science Direct, and Google Scholar) different combinations of some keywords (upper-limb, rehabilitation, robot, exoskeleton, shoulder, elbow, wrist, arm, therapy, assisted, training, stroke), moves through about 100 papers 


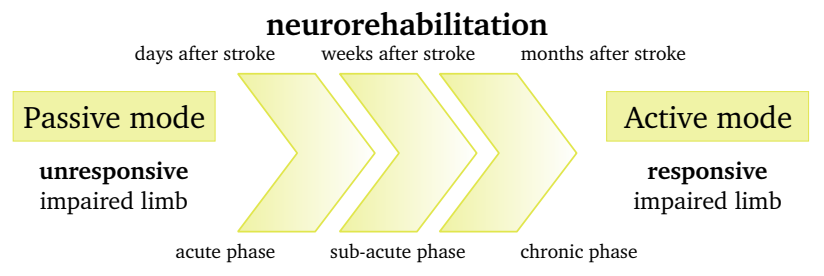

Fig. 1. General neurorehabilitation timeline. Robot controllers should adapt to the correct phase of the therapy of the stroke survivor. Passive and Active are traditional terms to indicate the involvement of the subject in the training.

published before March 2016. Besides, we also conducted a free search in the above-mentioned databases for references listed in keywords-based findings to include larger context. For a purpose of feasibility, we focused our analysis on the subset of minimum 3 degrees of freedom (DOF) actuated upper-limb exoskeletons for rehabilitation, with at least a control over two upper-limb joints among shoulder, elbow, and wrist. Besides, this decision reflects the idea of characterizing the available control strategies for multi-DOF devices, which apply forces along the assisted limb rather then acting only at the endeffector. Furthermore, a review including most of the planar robots is already available in literature [16]. In the following review, we briefly illustrate every available control solution, providing more details on the ones which were clinically tested.

\section{Available Control strategies}

32 robotic exoskeletons for upper-limb rehabilitation have been listed, considering only the devices with 3-DOF or more controlling the motions of minimum two out of the shoulder-elbow-wrist joint groups (see Table I). Four devices ([54], [55], [56], [57]) composed by multiple robots acting on multiple contact points were not included in this review. Among the 32 exoskeletons, to the authors' knowledge, 10 devices were tested on post-stroke impaired subjects, but only 6 test provided comparisons with impaired subject control groups which tested different rehabilitation therapies. Besides this, roughly one third of the existing structures seems not to be currently subject of study, or rather, no results are appearing for these devices on the main journals and conferences for at least three years.

Inspired by the categories presented in [16], we defined three main global strategies for robotic-mediated rehabilitation: assistance, correction and resistance, see figure 2.

- Assistance means that the robot is supporting the weight of the impaired limb and providing forces to complete the task. If the patient does not produce any effort, task completion can be still achieved depending on the level of assistance.

- Correction defines the rehabilitation situation in which the robot is only acting when the patient is not performing the movement correctly, forcing the impaired limb to recover a desired inter-joint coordination.

- Resistance represents the techniques in which the robot opposes forces to the motion (potentially increasing the current error, for example) in order to make the task more

\begin{tabular}{|c|c|c|c|c|c|c|c|}
\hline \multirow[b]{2}{*}{ project name } & \multirow[b]{2}{*}{ nationality } & \multirow[b]{2}{*}{ year } & \multicolumn{2}{|c|}{ DOF } & \multirow[b]{2}{*}{ type } & \multirow[b]{2}{*}{ pHRI } & \multirow{2}{*}{$\begin{array}{l}\text { clinica } \\
\text { testing }\end{array}$} \\
\hline & & & a & $\mathrm{p}$ & & & \\
\hline \multicolumn{8}{|c|}{ supported motion of shoulder-elbow-wrist } \\
\hline ARAMIS [17] & Italy & 2009 & 6 & 0 & $\mathrm{e}$ & 2-sfh & [17] \\
\hline ARMinIV [18] & Switzerland & 2007 & 7 & 0 & $\mathrm{e}$ & ufh & [19] \\
\hline ArmeoPower ${ }^{1}[11]$ & Switzerland & 2011 & 6 & 0 & $\mathrm{e}$ & ufh & 1 \\
\hline ARMOR [20] & Austria & 2008 & 8 & 4 & $\mathrm{e}$ & 2-uffh & {$[20]$} \\
\hline BONES+SUE [21] & USA & 2008 & 6 & 0 & $\mathrm{p}$ & ufh & [22] \\
\hline BOTAS [23] & Japan & 2013 & 6 & 0 & $\mathrm{e}$ & 2-ufh & \\
\hline ETS-MARSE [24] & Canada & 2010 & 7 & 0 & $\mathrm{e}$ & ufh & \\
\hline EXO-UL7 $^{2}[25]$ & USA & 2011 & 7 & 0 & $\mathrm{e}$ & 2-ufh & {$[25]$} \\
\hline IntelliArm [26] & USA & 2007 & 7 & 2 & $\mathrm{e}$ & ufh & \\
\hline NTUH-ARM [27] & Taiwan & 2010 & 7 & 2 & e & uf & \\
\hline RUPERT IV [28] & USA & 2005 & 5 & 0 & $\mathrm{p}$ & sufh & \\
\hline SRE [29] & UK & 2003 & 7 & 0 & $\mathrm{p}$ & fh & \\
\hline SUEFUL 7 [30] & Japan & 2009 & 7 & 1 & $\mathrm{e}$ & uffh & \\
\hline \multicolumn{8}{|c|}{ supported motion of shoulder-elbow } \\
\hline$-[31]$ & France & 2009 & 4 & 0 & $\mathrm{e}$ & uf & \\
\hline$-[32]$ & New Zealand & 2014 & 5 & 0 & $\mathrm{e}$ & uh & \\
\hline ABLE [33] & France & 2008 & 4 & 0 & $\mathrm{e}$ & uf & [34] \\
\hline ALEx [35] & Italy & 2013 & 4 & 2 & e & ufh & \\
\hline AssistOn-SE [36] & Turkey & 2012 & 6 & 1 & e & ufh & \\
\hline CAREX [37] & USA & 2009 & 5 & 0 & $\mathrm{e}$ & suf & \\
\hline CINVESRobot-1 [38] & Mexico & 2014 & 4 & 0 & $\mathrm{e}$ & uf & \\
\hline L-Exos [39] & Italy & 2002 & 4 & 1 & e & ufh & {$[40]$} \\
\hline LIMPACT [41] & Netherlands & 2008 & 4 & 6 & $\mathrm{~h}$ & uuff & \\
\hline MEDARM [42] & Canada & 2007 & 6 & 0 & $\mathrm{e}$ & uf & \\
\hline MGA [43] & USA & 2005 & 5 & 1 & $\mathrm{e}$ & uh & \\
\hline MULOS [44] & UK & 2001 & 5 & 0 & $\mathrm{e}$ & uff & \\
\hline Pneu-WREX [45] & USA & 2005 & 4 & 0 & $\mathrm{p}$ & ufh & {$[46]$} \\
\hline RehabExos [47] & Italy & 2009 & 4 & 1 & $\mathrm{e}$ & ufh & \\
\hline \multicolumn{8}{|c|}{ supported motion of elbow-wrist } \\
\hline 6-REXOS [48] & Sri Lanka & 2015 & 4 & 2 & $\mathrm{e}$ & fh & \\
\hline MAHI EXO-II [49] & USA & 2006 & 5 & 0 & $\mathrm{e}$ & ufh & 3 \\
\hline MAS [50] & Japan & 2008 & 4 & 0 & $\mathrm{p}$ & ufh & \\
\hline ULERD [51] & Japan & 2013 & 3 & 4 & $\mathrm{e}$ & uufh & \\
\hline Wrist Gimbal [52] & USA & 2013 & 3 & 0 & $\mathrm{e}$ & fh & \\
\hline
\end{tabular}

TABLE I

EXOSKELETONS FOR UPPER LIMB REHABILITATION (3-DOF SYSTEMS CONTROLLING AT LEAST TWO JOINTS OUT OF THE SHOULDER-ELBOW-WRIST CHAIN). DOF: A-ACTIVE THUS ACTUATED, P-PASSIVE THUS MECHANICAL ONLY. TyPe of aCtuation: E-Electrical, P-PNEUMATIC, H-Hydraulic. PhySiCAl HUMAN-ROBOt INTERFACE (FIXATION LEVELS) : 2-TWO ARM EXOSKELETON, S-SHOULDER, U-UPPER ARM, F-FOREARM, H-HANDLE. DOUBLE LETTERS INDICATES DOUBLE INTERFACES. ${ }^{1}$ BASED ON ARMINII, THE ONLY COMMERCIALIZED EXOSKELETON FOR THE CLINICAL ENVIRONMENT. ${ }^{2}$ BASED ON CADEN-7 (2006)

[53]. ${ }^{3}$ ONGOING Test, ClinicalTrials.gOV identifier: NCT01948739.

complex for the subject, and to train his ability to correct the movement and to adapt to external perturbations.

Contrary to assistance, correction does not assist the patient in achieving the task. Obviously, pure correction is an ideal case of neurorehabilitation therapy, as well as the former categorization. More often, the therapy involves several strategies combined [10].

While resistive controllers exist for manipulanda (for example, resistive force-field in [58], or error augmentation in [59]), to the authors' knowledge, to date there are no available resistive controllers developed for exoskeletons. This could be a consequence of the fact that exoskeletons often target early stage chronic patients who rarely have recovered enough motor capabilities to undergo resistive therapies. However, most control strategies developed for manipulanda could be translated to exoskeletons, by considering only the end-effector control; though this solution would not take the full advantages of dealing with multi-contact systems like the exoskeletons.

Existing controllers for exoskeletons are mostly combined 


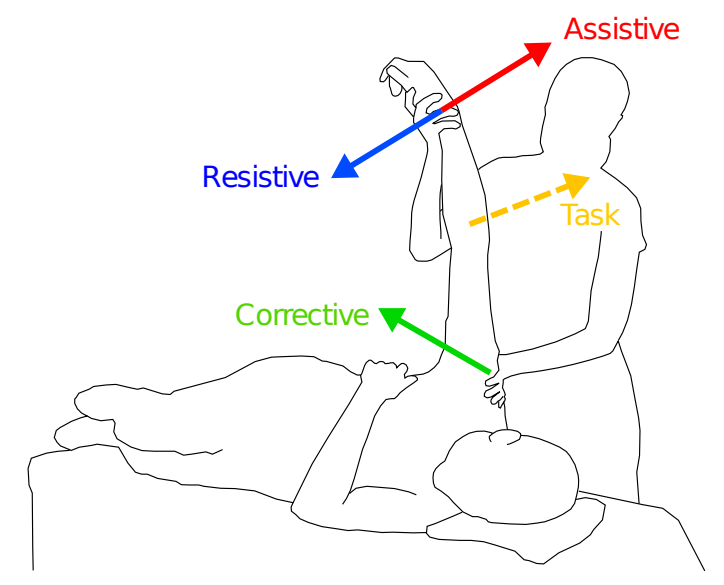

\author{
Assistive mode \\ 1. Passive control \\ Passive trajectory tracking, Passive mirroring, Passive stretching \\ 2. Triggered passive control \\ 3. Partially assistive control \\ Impedance/Admittance control, Attractive force-field, \\ Model-based assistance, Offline adaptive control

\section{Corrective mode} \\ 1. Tunneling \\ 2. Coordination control
}

\title{
Resistive mode
}

no controls developed yet

Fig. 2. The three global strategies for robotic-mediated rehabilitation and the current implementations on robotic exoskeletons.

assistive-corrective controllers but there exists a variety of implementations of each category by using different control techniques.

\section{A. Assistive modes}

Within this type of control approaches, three groups of assistive strategies were determined - passive, triggered passive, and partially assistive control - although there exist solutions which are mixed strategies. The distinction between passive and partially assistive controls is thin, since if the patient is not participating in the task, both controllers would react similarly. Also, triggered assistance generally refers to solution only different to initiate the assistance: once triggered, these controllers usually exhibit either passive or partially assistive behaviours.

1) Passive control: The simplest way to control an exoskeleton is to control the motion rigidly along a desired reference trajectory through position feedback control with high corrective gains. In rehabilitation this passive technique is common at early stages of the post-stroke therapy, when the impaired limb is usually unresponsive, and thus passive mobilization is the only feasible solution to achieve any result. Nonetheless the feedback controller gains have to be tuned carefully and the exoskeleton has to show a minimum of compliance not to hurt the subject in the presence of trajectory errors due to excessive muscle contraction, spasticity, or other pathological synergies. Such compliance between the robot and the human body can also be introduced mechanically, at the fixation level with, for example, elastic straps [28] or mechanical fuses placed serially [57].

$\triangleright$ Passive trajectory tracking: Passive control can be achieved by adopting different techniques. The simplest is the use of a proportional-integral-derivative (PID) feedback control which usually regulates the position or the interaction force along a known reference (for example, a trajectory or a force field model), and can be applied either at the joint or at the end-effector level. Examples of these joint controllers are shown in [38], [52], [60], [43], [61], [47], [62], [41].

More complex approaches have been recently developed to improve the quality of the physical interaction during passive mobilization of patient's limb. In [24], the authors developed the Sliding Mode Control with Exponential Reaching Law (SMERL), a non-linear control technique which minimizes the tracking error on a state space projection. In [63] a fuzzy logic technique is adopted. In order to deal with uncertainties and disturbances from the environment, adaptive fuzzy approximators estimated the dynamical uncertainties of the human-robot system, and an iterative learning scheme was utilized to compensate for unknown time-varying periodic disturbances. Preliminary results on healthy people showed better performances, in terms of tracking error and average control input, compared to classical PID control and fuzzy logic techniques used alone.

Different methods exist for defining reference trajectories. For passive strategies, these references are often created by recording the physiotherapist inputs on the subject limb attached to the exoskeleton, during a teaching phase. In this phase, the robot is generally set in a transparent mode (usually achieved by adopting a feedforward term to compensate for the gravity and the dynamics of the robot) to limit any resistance to perform motions. Once recorded, the exoskeleton is ready to replay the trajectory with its feedback controller. Teach-andreplay is presented in [64]. In [60] teach-and-replay mode was tested on four chronic stroke patients during 8 weeks of training. Encouraging preliminary results were obtained (increase of Fugl-Meyer Assessment score - FMA, meaning improvement of motor functionality of the paretic arms, in addition to positive transfer to ADLs). Nevertheless, no comparisons with control group receiving traditional therapy were provided. Similarly to teach-and-replay, record-and-replay uses healthy limb motions, recorded within the robot, to create reference trajectories. This strategy was for example used in [17].

Instead of using external inputs or "healthy" trajectories as references, some research groups have been trying to directly detect patient motion intention by measuring muscle activity through surface-EMG. In [65] EMG and two offlinetrained time delayed neural networks (one for the shoulder and one for the elbow) were used to estimate and predict the resulting torques at the joint level. By using this prediction, a reference joint position trajectory was computed and fed to a PD controller with gravity and friction compensation. In [66] 
an EMG-based version of the SMERL controller was provided. In this case, EMG signals, transformed to a desired position reference through a muscle model, acted as the reference for the position feedback controller.

A third strategy to define reference trajectories is to determine cost functions and to use optimization algorithms. Generally the cost function aims at minimizing the jerk. This solution is mostly used for triggered passive control (for example in [23]) or for partially assistive controls (examples in [67] or [68]). Due to the complexity and variety of possible actions of the upper-limb, generally the movements of intermediate joints either occur as a consequence of the endeffector movement in the task space (for example in [37]), or are constrained along specific dedicated trajectories, which are synchronized within the joint space (for example in [69]). However, because of the redundancy of human arm, there are no commonly accepted solution to compute joint trajectories from task space motions [70].

Beside standard passive control approach, two other passive strategies exist. Those either target two-arm exoskeletons (passive mirroring) or utilize a different rehabilitative training, i.e. the passive stretching.

$\triangleright$ Passive mirroring: Few exoskeletons have two arms (four devices in Table I, column pHRI). For these robots, passive mirroring is a further strategy. It consists of synchronous passive mimicking of the behaviour of the healthy limb, in a master-slave configuration, as in [17] or in [20]. In [17] a clinical pilot study on 14 impaired subjects ( $21 \pm 6$ days since stroke), based on an average of 31 sessions over 2 months, resulted in applicability and tolerability of the robot-mediated treatment, with similar improvements to other robot-assisted rehabilitation therapies. In [71] passive-mirroring was implemented on a single-arm robot in a master-slave configuration with an external haptic device to control the master side of the system.

$\triangle$ Passive stretching: A different rehabilitative passive training is the so-called passive stretching [26]. With this strategy, individual joints were passively stretched by the robot in order to identify their individual angle-resistance torque relationships. These relationships were then used to coordinate the passive stretching of multiple joints together. Feasibility test performed in 3 stroke patients showed a reduction of crosscoupled stiffness after a 40 min stretching session.

2) Triggered passive control: A slight variation of assistive modes consists in approaches in which the user triggers the exoskeleton assistance. This technique is frequently used to introduce brain-machine interface (BMI) into the control loop. These approaches are directly adapted from the field of assistance to patients with non-recoverable impairments (like tetraplegia). In fact, after the triggering (mainly a selection of available targets), the exoskeleton is usually controlled passively along pre-determined trajectories.

In [23] passive recorded-trajectory replication through BMI control is shown. The end-effector trajectory were computed by minimum-jerk optimization. BMI trajectory-replay triggering was implemented through SSVEP (steady-state visual evoked potential). SSVEP can be observed mainly from the visual cortex when a person is focusing his visual attention on a flickering stimulus. Pre-clinical testing (12 healthy and 3 upper cervical spinal cord injured subjects) were performed to show the capacity of the system to be used by subjects to activate different trajectory reproductions.

In order to extend patient's control over the task, gaze tracking methods can also be used in addition to BCI-driven control like in [72]. An eye-tracker together with a target-tracking module (a Kinect camera) gave the reference position of the target to reach to the exoskeleton controller; the BCI module estimated user's motion intention, modulating maximum joint jerk, acceleration, and speed within an admissible predefined set of values. Then a PD feedback control (helped by classical gravity and friction compensations) performed the reaching task. Pre-clinical testing with 3 healthy and 4 chronic stroke patients showed that all subjects were able to operate with the exoskeleton.

In [73] a Motor Imagery based Brain Computer Interface (MI-BCI) is used to control an eight DOF exoskeleton. Three chronic stroke survivors were able to perform passive controlled tasks (arm motion towards a target, grasping and releasing of an object) by producing MI of the reaching task before the movement. Two seconds of MI activity collected, triggered the exoskeleton to replay pre-recorded trajectories. In [74] authors developed a similar approach, by controlling an ArmeoPower exoskeleton through MI-BCI. 9 healthy subjects and 2 stroke survivors were able to move the exoskeleton along pre-defined trajectories, and these motions were brain-state dependent, meaning that motion was performed only during MI phases.

3) Partially assistive control: The effectiveness of pure passive motions for stimulating neuroplasticity is known to be limited [75], since the patient is not involved in making any effort to perform the task. On the other side, assistance is needed in order to reduce failures at least at the beginning of the therapy, thus maintaining subject motivation, intensity of the training, confidence in using the affected limb, and to avoid negative reinforcement [6]. But as soon as the patient has recovered a minimal amount of motor capacity, it is essential for the robot to allow for shared control of the movements [76], [77]. Indeed, as neurorehabilitation addresses issues related to motor control, the devices must allow patients to express whatever movement they can without suppressing any motor capability [78].

Partial assistance, or assistance-as-needed [67], groups all those control strategies which allow the impaired subject to actively control the motion, supporting it based on performance indexes. The most common solution to provide a partial assistance to the impaired limb, is to increase the compliance of the above-mentioned passive controllers. Instead of rigid "industrial-type" position feedback controllers (with high corrective gains), many controllers for exoskeletons rely on more flexible impedance control [79], or its dual admittance control, with reduced corrective gains to exhibit "human-like" mechanical properties. These controllers allow to implement a good compromise between tracking skills and compliance of the robotic arm.

$\triangleright$ Impedance/Admittance control: Impedance control is a model-based force controller with position feedback while its 
dual, the admittance control, is a position controller with force feedback. Impedance control is efficient for lightweight backdrivable exoskeletons (often cable-driven devices). In these systems, problems arise when it is necessary to compensate for gravity and friction. For exoskeletons that lack backdrivability, admittance control may be more appropriate, because the forces at the interfaces with the human limb must be measured to move the robot, thus considering its inertia and dynamic effects. The force-tracking trade-off feature, together with the simplicity of implementation, are the reasons why these are two of the most common control algorithms currently used for rehabilitation exoskeletons [49], [64], [43], [80], [60], [81].

Adopting an admittance/impedance approach, some research groups developed slightly different and more complex strategies. In [82] the exoskeleton was controlled through two impedance control loops, one for assistance along the endeffector reference trajectory, the other to apply corrections along the orthogonal direction to the trajectory. Model-based gravity compensation was also present. The setup was supported by a virtual environment on which a reaching game was created. A therapist could directly tune tasks complexity on a therapy control unit. Pre-clinical testing (8 post-stroke subjects), three one-hour rehabilitation sessions per week for a total of six weeks, resulted in an increase of performances and a reduction of position errors after the therapy for 5 patients. The same controller was tested in [40] on 9 impaired subjects performing three games (pointing task, circle drawing, and puzzle-type game). The robot-aided training induced, independently from the post-stroke phase, statistical significant improvements of kinesiologic and clinical parameters (such as analysis of reaching movements, degree of functional impairment through FMA, active range of motion, and Modified Ashworth scale), as a result of the increased active ranges of motion and improved co-contraction index for shoulder extension/flexion.

In [83], the authors developed a model-free PID type admittance control in task space to generate desired trajectories for a lower-level PID position control in task space too. The control parameters can be chosen based on kinematics and dynamics of the human arm. Besides, a simplified model-based gravity compensation was used. In [84] the authors compared task space with joint space admittance control, showing that task space control spent about $11 \%$ less of interaction energy for a peg-in-hole task. In [30] the exoskeleton was controlled by an end-effector impedance control. A joint torque fuzzy logic estimator (using signals from 16 sEMG channels and a couple of force/torque sensors) evaluated the user hand motion intention. The fuzzy set of membership functions determined which data considering from a combination of the two inputs: if the EMG values were high, the estimation was only EMGbased; if they were low, it was only force/torque based; otherwise a continuous mixed integration of both signals was adopted. In [51] admittance control was computed by substituting force sensors with springs, and their compression measured to estimate interaction force between the human limb and the robotic device. Two control techniques were implemented: respectively high stiffness mode when passive DOFs of the arm (a group of 4 springs) were locked, and resistive training when they were unlocked.

Apart from impedance-admittance controls, there exist others examples of assistive controls in the literature. In particular, we can describe three categories of assistance respectively based on attractive force-fields, on human arm model, and on offline adaptation.

$\triangleright$ Attractive force-field: [85] used, among other approaches, an attractive force field to control their exoskeleton during a clinical rehabilitation therapy with 15 impaired subjects. During the trial, subjects were randomly assigned to bilateral training (BT), unilateral training (UT), and standard therapy (ST) and executed a total of 12 sessions of virtual reality gaming (8 different games in total). While in BT partially assisted mirroring of the unaffected limb was provided by the robotic exoskeleton, in the UT an attractive force-field acting only on the end-effector was determined based on the endeffector position. For the other joints of the robot, an assistive force was produced based on reference trajectories computed by using the swivel angle estimation algorithm [86]. Following these treatments all of the subjects significantly improved their fine motor control and gross control across all the treatment modalities. BT showed better results compared to UT but this might have been because of more assistance from the robot to the impaired limb. However robot-mediated therapy exhibited comparable outcomes than standard therapy.

$\triangleright$ Model-based assistance: In [50], after an offline posture measurement through motion capture, authors derived a muscle force estimation to maintain a desired pose, by using a musculoskeletal human model. The reference muscle force was then fed into the device controller together with the current force, estimated through 3 EMG sensors, and a proportional control drove the pneumatic actuators to provide only the necessary force. In [87] the former method was improved to reduce non-target muscle efforts through an optimization algorithm, and tested for controlling quasi-static posture-by-posture human motion.

$\triangleright$ Offline adaptive control: Adaptation trial-by-trial is also a solution to modulate robot assistance based on different performance indexes. In [28] the authors implemented an iterative learning control: a feedforward assistive term was learnt using trial-by-trial adaptation, based on the error of the previous trial and on a fuzzy logic based non-linear function of the tracking error statistics. The feedforward term was then added to a PID feedback controller at the joint level. Preclinical testing with 6 impaired subjects to test its usability are shown in [88].

In [67] a PD controller was also used with a feedforward assistive term, which was adapted during the motion depending on the dynamics of the patients extremity, the patients neurological ability, and the patients effort, modelled through a Gaussian radial basis function based neural network. Assistance-as-needed was achieved by adding a force decay term to the adaptive control law, which reduced the output from the robot when errors in task execution were small. The controller was tested with 11 chronic post-stroke patients. Results interestingly shown the "slacking" behaviour of the human motor control, meaning that, as the central nervous system (CNS) tries to optimizes its efforts, as soon as it can 
take advantages of the controller, it leaves the controller taking over most of the physical effort required to complete the task. By adding the force decay term, the controller was able to encourage the patient efforts.

Two different tests of the same controller on two different exoskeletons were presented in [46] (clinical testing with 27 impaired subjects) and [22] (20 subjects with mild to moderate chronic stroke). In the first study, the subjects, divided into two groups, received either the assist-as-needed robot training, or conventional table top therapy with the supervision of a physical therapist. The results were moderately better by assisting the tasks with an assist-as-needed controller. In the second study, the subjects experienced multi-joint functional training and single joint training, generally improving motor function after chronic stroke. Multi-joint functional robotic training was anyway not decisively superior to single joint robotic training.

In [89] an adaptive PD controller, acting at the joint level, together with a model-based feedforward gravity compensation, regulated the stiffness of a robotic exoskeleton. Tracking error of the reference trajectory drove the trial-by-trial adaptation of the feedback gains. In [90] a sensorless force estimator, based on a Kalman filter, was used to dynamically determine the exoskeleton operator's capability performing the desired task, in order to provide him minimal assistance with an user-specified maximum allowable bounds on position error. Both the two former controllers were only preliminary tested on healthy subjects, showing the applicability of the two approaches.

Finally, in [91] an assistive-as-needed control algorithm tried to anticipate patients deviations from desired reference trajectories, by utilizing an adaptive model of the patient's dysfunctionality. Such a dysfunctional profile of the patient should be provided by the therapist, depending on both the specific ADL and the patient motion deficits. The anticipatory behaviour of the controller was only tested through a simulation in virtual reality by the authors.

\section{B. Corrective modes}

As described in the former section, many assistive controllers act on the trajectory followed by the subject not only in the direction of the desired motion, but also applying forces along the orthogonal direction, which is the domain of corrective control.

A key to understand the differences between the two approaches is the capability to complete successfully a motion without any effort from the subject: assistance is generally able to move the limb towards the final target, if the subject does not participate, while ideal corrective strategies cannot.

A similar concept can be expressed by considering the time-dependency of the references. Assistive controller are usually fed with desired trajectories, thus time-dependent speed profiles. Pure corrective strategy is linked to the idea of time-independence of the references, for example feeding the controllers with desired path instead of trajectory. The time-independence allows the robot to only act on the current position to correct it along the orthogonal direction.
However, it is often difficult to make a clear distinction between pure assistive control laws and pure corrective ones. Currently there exist two approaches which provide pure correction, or at least, in which assistance is clearly decoupled from correction.

1) Tunneling: Tunnelling consists in creating virtual channels for the end-effector or the joints of the exoskeleton, in which the subject moves: once he/she goes out of the channels, the feedback control takes him back into the channel, as if a spring impedance was attached from the limb to the center of the virtual channel. In addition, to prevent the subject from getting stuck during the motion, a supporting force in the direction of the channel is generally added. Therefore, tunneling strategies can be seen as an impedance control with a path-centred no-action zone, i.e. one can clearly consider the supporting force field as an assistive term, and the springdamper-like force in the direction of the centre of the channel as a corrective term.

In [18] the channel was acting on the end-effector position and the supporting force field was adapted depending on minimally desired mean velocity of the subject's limb. The system was also fed with control inputs from the gravity and friction feedforward compensator. The reference trajectory was divided in subtrajectories, and a trajectory generator algorithm updated the tunnel direction every time the subject achieved a subtask. Pre-clinical testing on healthy and 3 impaired subjects showed the feasibility of such control paradigm. The same controller was tested in [19] in a clinical experiment with 77 stroke survivors. Various games and ADLs were tested in a virtual reality environment, augmented with online audiovisual information to increase subjects motivation. After 8 weeks of training, small improvements in FMA scores were higher in patients assigned to robotic therapy than in those undergone conventional therapy. A similar approach was adapted by [92]. Due to the cable-driven system, simultaneously to the highlevel tunnelling controller, a low-level PI plus feedforward control kept the tension of the cables [93]. Pre-clinical testing on 8 healthy and 1 impaired subject, showed that the subjects constantly moved closer to a prescribed path in the training trials.

2) Coordination control: When considering corrective modes, the aim of the control law is generally to regulate the coordination (also called "synergies" [94]) during the movement, i.e. controlling joint positions and/or velocities relative to the others. Trying to coordinate inter-joint positions or speeds is a way to solve the reference time-dependency problem, which remain a constraining parameter of the assistive controllers.

In [95] the time-independent functional training (TIFT) algorithm was introduced: virtual joint-space walls kept the subject close to the ideal joint-space path, acting simultaneously on multiple joints motion as PD position controls. This strategy provided assistance as correction to bad motion coordination of the main joints active during the task, in particular on shoulder and elbow joints. This controller was tested on 12 impaired subjects in a 3-month pilot study [96]. Data suggested that this robotic therapy could elicit improvements in arm function that were distinct from conventional therapy 
(improved inter-joint coordination), and thus it could integrate to conventional methods to improve outcomes.

In [34] an alternative approach was presented, the Kinematic Synergy Control (KSC). In this work, the problem of tracking was projected from the reference trajectory space to the joint velocities synergy space through Principal Component Analysis (PCA). The exoskeleton generated reactive viscous joint torques to impose specific patterns of interjoint coordination without constraining the hand motion. This controller was tested with healthy subjects and, preliminarily, with hemiparetic patients, and showed its the ability to impose constrained synergies without altering the hand motion.

[97] developed an approach based on correction of the pathological involuntary flexion torque, which occurred at the elbow during shoulder abduction. During an evaluation session with the robot, the patients pathological involuntary torque was measured and a counter-active, just-as-needed torque was then calculated and applied during the therapy. A case study on one patient showed a reduction of elbow involuntary elbow flexor torque during shoulder abduction. [26] used a similar approach to develop a passive stretching controller for multiple joints. However no active modes of therapy, during which the subject is actively participating in the movement, based on previously identified angle-torque relationships, have been developed to target patterns of inter-joint coordination.

\section{DISCUSSION}

In this paper, we reviewed the currently available control strategies for robotic exoskeletons for neurorehabilitation, among about 100 publications and about 30 existing devices. This review allowed us to spot several issues and scientific barriers potentially limiting - to the authors' opinion - the rehabilitative performances of current exoskeleton devices. In the following section, we discuss some of these analyses.

\section{A. Hardware limiting control possibilities}

One of the performance indexes that quantifies an exoskeleton ability to precisely produce a programmed assistance to the subject, is its transparency. This may seem contradictory, since transparency measures the robot ability of not applying any assistance/resistance to free motion. But this property is a good indicator for force precision, since any failure to reach transparency during a zero-resistance experiment will be reproduced and act as a bias in a non-zero force experiment.

Transparency therefore qualifies the mechanical properties of the structure (weight, inertia, friction, etc.), of its actuation (backdriveability, friction, etc.), and of the performances of the low level control dedicated to the compensations of these perturbing phenomena (feedforward compensation of the gravity and friction, for example).

A lack in transparency can generate undesired resistances during the upper-limb motion of subjects (even healthy), from which excessive muscle efforts can arise to complete even simple motions. This, obviously, can be negligible for healthy subjects, even if it is already highly perturbing [98], but it cannot be allowed with impaired patients during rehabilitation. Moreover the lack of transparency can make almost impossible to develop pure corrective strategies on the exoskeletons, since it does not really allow shared control (the human on the motion, the controller on the corrective part).

The transparency level directly influences the physical behaviour of the robot, and possibly perturbs the rehabilitative control strategy of the robot. Therefore it would be necessary to assess exoskeletons standard features. For example, simply showing comparisons of motion ability (like range of motion) of healthy subjects with and without the exoskeleton, almost always lacks in exoskeleton design papers. Such an analysis was recently performed on the only commercially available exoskeleton for neurorehabilitation, and shows that it significantly affects the reaching movements of healthy subjects [99].

Clinical results obtained with any platform are possibly not qualifying the rehabilitation capacity of a control strategy but rather the global performance of the system. A specific care should therefore be given to draw general conclusions on rehabiltiation approaches with exoskeletons.

Similarly, while researchers are starting to consider exoskeletons sensors as a direct source of data to evaluate patient state and recovery evolution [100], it is important to highlight the fact that the capability of modern exoskeletons to provide correct assessment of quality of human motion, directly lies on their ability to be transparent.

\section{B. The problem of reference definition}

As showed in section II, a central issue for most research groups is related to the generation of healthy reference trajectories and thus, to the computation of error profiles to supply the feedback controllers. Physiotherapists also share the necessity of reference for applying correct torque profiles on the impaired arm and, more generally, evaluating subject movements. With the experience, they learn how to correctly manipulate impaired limbs to induce neuroplasticity and recover motor control. Translating this qualitative feature into a quantitative reference for the robotic devices is a key issue, especially for $3 \mathrm{D}$ movements when considering interaction at the joint level.

The reference generation is a two-level problem. At the low level, the exoskeleton must be fed with human arm suited feasible trajectories to successfully complete the desired task, but taking into account the natural redundancy of human arms, at the high level, the issue is to select one of the many correct possibilities to perform the motions and achieve the task. Additionally, the ideal reference trajectory should be customized on the patient features, taking care of the specific arm physical characteristics and impairments, such as paralysis, spasticity, and joint limitations.

It is important to underline how the reference generation is a different problem from controlling the exoskeleton. Indeed, the same controller may lead to different rehabilitative performances being fed with references computed by using different techniques.

In section II, we described mainly three different approaches for generating motion references. The simplest technique is about directly recording the motion of the human limb (teaching/recording). This generation process of recording and extracting a trajectory is time-consuming and not generalizable to 
multiple tasks, but it produces always feasible and customized references.

A second common technique is about using (optimization algorithms). Computational models usually exploit minimum jerk optimization to generate the desired smooth feasible trajectory. This optimization technique has its advantages in the quickness of providing a solution, but this solution is still valid for one-single task.

The last technique is about the possibility of anticipating the human CNS by detecting motion intentions, or more simply by detecting, in a set of predefined trajectories, the one to be performed (motion intention detection). This approach is spreading as a common solution for many research groups, even if there exist generic technical issues concerning, for example, discrete signal extraction (either from the brain or from the muscles), noise deletion, etc. Motion intention detection can be realized through force/torque sensors (for example in [101]), EMG ([65] and [30]), or through oculography and EEG ([72] and [23]). The resulting controllers appear often to be passive-triggered controllers which replay pre-determined reference trajectories either recorded or computed through optimization algorithms.

A fourth solution to produce motion models is the generation of feasible trajectories through the evaluation of statistically consistent patterns performed by a sufficient number of healthy subjects. Nonetheless, due to its complexity, this technique is not commonly used for rehabilitation robotics and only one example can be found in [91].

Despite the above-mentioned strategies, it is important to underline that relying on a reference trajectory limits the efficacy of the rehabilitation therapy since these trajectories are generally position and time dependent and, therefore, they are complex to generalize for different movements, targets, or tasks. This means that the patients freedom of movement, within the exoskeleton, is limited to specific movements. These mode of interaction constantly imposing constraints (more or less rigidly) is less physiological than conventional therapy (like, for example, the Bobath therapy, during which the patient is active, occasionally guided or corrected, but not constantly constrained to follow a fixed gesture) and therefore its efficiency is questionable.

In order to increase human involvement in the rehabilitative therapy, thus increasing the efficacy of the therapy itself, exoskeletons controllers should target goal-independent strategies. Ideal goal-independence is a solution which allows multiple targets reaching and tasks achievement, without acting on the controller, neither the control law nor the reference. Thus, in this case, the reference is necessary described at a higher level of abstraction than the simpler common trajectory reference. Together with goal-independence, time independence, as defining path references rather than trajectories, is a key factor to allow the subject to actively make an effort to achieve the task, not being constrained by following rigid planned trajectories.

\section{Addressing coordination control}

Time independence translates into a necessity to develop inter-joint/coordination controllers, more than trajectory track- ers. In fact, this kind of controllers requires relative references (for example, speed profiles of joint synergy coordination) more than absolute ones, as, for example, joint positions profiles in the case of trajectories. The importance of measuring and controlling joint synergy is primary to better understand quality of movements during rehabilitative therapy. Post-stroke patients frequently adopt negative compensatory strategies that are difficult to be analysed without coordination assessment. In [100] authors review current assessment strategies for motor recovery with robotic exoskeletons after stroke. Their emblematic conclusion says that most assessments for roboticmediated therapy addresses end-point movements, ending in a minimal measure of compensatory joint synergy strategies. Without the latter, it is complex to distinguish between genuine motor recovery and dangerous compensation.

Few research groups developed solutions aiming at controlling synergy joint coordination [97], [26], [95], [34], as described in section II-B2. However, for all these approaches, only preliminary results exist.

\section{Clinical testing issues}

What arises from the general overview of this state of the art and of Table II, is the limited number of studies and experiments both on healthy and impaired subjects. Indeed, among all the devices, only 8 of them were clinically tested, i.e. providing comparisons between different developed control strategies and/or standard therapies, with impaired subject control groups. Furthermore, only three studies [19], [46], and [22], involved 20 or more stroke survivors. For all these studies, robotic-mediated therapy generally produced small improvements in impairments measures and motor recovery compared to standard therapy on the same number of repetitions. This improvements were usually detected by FMA, range of motion tests, and measurements of strength. Only one study [22] described functional improvements after robotic training. Clearly, neither motor recovery nor functional improvement alone necessary translate to improvements in ADLs or in quality of motions, since there may still be unhealthy compensations (in case of functional improvements) or no reallife relevance (in case of motor recovery) [10].

A problem arising is the absence of a standard assessment method of the results of the robotic-mediated therapy, especially with exoskeletons (i.e. with multiple interaction points): the chosen kinematic parameters specify too often end-point movements only, and are not able to address directly motion coordination. Moreover, these parameters show insufficient correlation with standard clinical assessment [100], thus they are difficult to be used as predictor to the clinical outcomes of robot-mediated therapy.

However, it is important to underline that each single clinical result is strictly linked to the device and the selected control strategy. In fact, every test is the result of a combination of the mechanical properties of the available exoskeleton, its transparency level, the chosen control strategy, and the type of generated reference. Thus, it is yet difficult to produce generic conclusions about the results of exoskeleton-mediated therapy. 


\begin{tabular}{|c|c|c|c|c|c|}
\hline Ref. & Device & Year & No. of subjects & Control strategy & Summary of the results \\
\hline$[19]^{*}$ & ARMin III & 2014 & 77 & Tunneling & Small improvements in FMA score \\
\hline$[46]^{*}$ & Pneu-WREX & 2012 & 26 & Assistance-as-needed & Small improvements in FMA score \\
\hline$[22]^{*}$ & BONES & 2013 & 20 & Assistance-as-needed & $\begin{array}{l}\text { Improvements in FMA and functional tests (like } \\
\text { WMFT), no differences between multi-joint/single- } \\
\text { joint training }\end{array}$ \\
\hline$[85]^{*}$ & EXO-UL7 & 2013 & 15 & $\begin{array}{lr}\text { BT } & \text { (mirroring), } \\
\text { and } & \text { UT } \\
\text { assistance) }\end{array}$ & $\begin{array}{l}\text { Improvements in score of few efficency indexes } \\
\text { but small improvements in FMA score, BT better } \\
\text { performing than UT }\end{array}$ \\
\hline$[17]^{*}$ & ARAMIS & 2012 & 14 & Rec-and-Replay & $\begin{array}{l}\text { Proof-of-concept test, similar score as other test with } \\
\text { exoskeletons }\end{array}$ \\
\hline$[40]^{*}$ & L-Exos & 2012 & 9 & Partial assistance & Small improvements in FMA score \\
\hline$[88]$ & RUPERT IV & 2011 & 8 & Passive & Feasibility test \\
\hline$[34]^{*}$ & ABLE & 2012 & 7 & Coordination control & $\begin{array}{l}\text { Preliminary results on improvements on shoulder- } \\
\text { elbow coordination }\end{array}$ \\
\hline$[25]^{*}$ & EXO-UL7 & 2013 & 5 & $\begin{array}{l}\text { BT (mirroring), and } \\
\text { UT (transparency) }\end{array}$ & $\begin{array}{l}\text { Same improvements as standard therapy, UT less } \\
\text { performing than BT }\end{array}$ \\
\hline$[26]^{*}$ & IntelliArm & 2013 & 3 & Passive stretching & Reduction of cross-coupled stiffness \\
\hline$[23]$ & BOTAS & 2013 & 3 & BMI & Feasibility test \\
\hline [92] & CAREX & 2014 & 1 & Tunneling & Preliminary results on better trajectory tracking \\
\hline
\end{tabular}

TABLE II

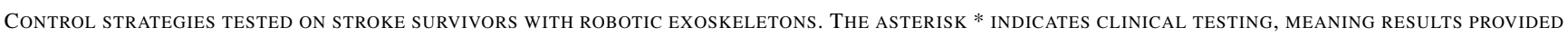
COMPARING DIFFERENT CONTROL STRATEGIES.

\section{REFERENCES}

[1] "World health organization website," Mar. 2013.

[2] A. Alwan et al., Global status report on noncommunicable diseases 2010. World Health Organization, 2011.

[3] C. Richards, F. Malouin, and S. Nadeau, "Chapter 13 - stroke rehabilitation: clinical picture, assessment, and therapeutic challenge," in Sensorimotor RehabilitationAt the Crossroads of Basic and Clinical Sciences (S. N. Numa Dancause and S. Rossignol, eds.), vol. 218 of Progress in Brain Research, pp. 253 - 280, Elsevier, 2015.

[4] M. Lotze, C. Braun, N. Birbaumer, S. Anders, and L. Cohen, "Motor learning elicited by voluntary drive," Brain, vol. 126, no. 4, pp. 866872,2003

[5] N. Schweighofer, Y. Choi, C. Winstein, and J. Gordon, "Task-oriented rehabilitation robotics," Am J Phys Med Rehabil, vol. 91, no. 11, pp. S270-S279, 2012.

[6] C. J. Winstein and D. Kay, "Chapter 16 - translating the science into practice: shaping rehabilitation practice to enhance recovery after brain damage," in Sensorimotor Rehabilitation At the Crossroads of Basic and Clinical Sciences, vol. 218 of Progress in Brain Research, pp. 331 - 360, Elsevier, 2015.

[7] G. Kwakkel, B. Kollen, and H. Krebs, "Effects of robot-assisted therapy on upper limb recovery after stroke: a systematic review," NNR, 2007.

[8] B. Volpe, H. Krebs, N. Hogan, L. Edelsteinn, C. Diels, and M. Aisen, "Robot training enhanced motor outcome in patients with stroke maintained over 3 years," Neurology, vol. 53, no. 8, pp. 1874-1874, 1999.

[9] A. Lo, P. Guarino, L. Richards, J. Haselkorn, G. Wittenberg, D. Federman, R. Ringer, T. Wagner, H. Krebs, B. Volpe, et al., "Robot-assisted therapy for long-term upper-limb impairment after stroke," $N$. Engl. J. Med., vol. 362, no. 19, pp. 1772-1783, 2010.

[10] V. Huang and J. Krakauer, "Robotic neurorehabilitation: a computational motor learning perspective," JNER, vol. 6, no. 1, p. 5, 2009.

[11] R. Riener, M. Guidali, U. Keller, A. Duschau-Wicke, V. Klamroth, and T. Nef, "Transferring armin to the clinics and industry," Top Spinal Cord Inj Rehabil, vol. 17, pp. 54-59, May 2011.

[12] P. Maciejasz, J. Eschweiler, K. Gerlach-Hahn, A. Jansen-Troy, and S. Leonhardt, "A survey on robotic devices for upper limb rehabilitation," JNER, vol. 11, p. 3, Jan. 2014.

[13] W. Chang and Y. Kim, "Robot-assisted therapy in stroke rehabilitation," Int J Stroke, vol. 15, no. 3, pp. 174-181, 2013.
[14] H. Lo and S. Xie, "Exoskeleton robots for upper-limb rehabilitation: State of the art and future prospects," MED ENG PHYS, vol. 34, pp. 261-268, Apr. 2012.

[15] L. Marchal-Crespo and D. Reinkensmeyer, "Review of control strategies for robotic movement training after neurologic injury," JNER, vol. 6 , no. 1 , p. $20,2009$.

[16] A. Basteris, S. Nijenhuis, A. Stienen, J. Buurke, G. Prange, and F. Amirabdollahian, "Training modalities in robot-mediated upper limb rehabilitation in stroke: a framework for classification based on a systematic review," JNER, vol. 11, no. 1, p. 111, 2014.

[17] L. Pignolo, G. Dolce, G. Basta, L. Lucca, S. Serra, and W. Sannita, "Upper limb rehabilitation after stroke: Aramis a robo-mechatronic innovative approach and prototype," in BioRob, pp. 1410-1414, IEEE, 2012.

[18] M. Guidali, A. Duschau-Wicke, S. Broggi, V. Klamroth-Marganska, T. Nef, and R. Riener, "A robotic system to train activities of daily living in a virtual environment," MED BIOL ENG COMPUT, vol. 49, pp. 1213-1223, July 2011.

[19] V. Klamroth-Marganska, J. Blanco, K. Campen, A. Curt, V. Dietz, T. Ettlin, M. Felder, B. Fellinghauer, M. Guidali, and A. Kollmar, "Three-dimensional, task-specific robot therapy of the arm after stroke: a multicentre, parallel-group randomised trial," Lancet Neurol, vol. 13 , no. 2, pp. 159-166, 2014.

[20] A. Mayr, M. Kofler, and L. Saltuari, "Armor: Elektromechanischer roboter fr das bewegungstraining der oberen extremitt nach schlaganfall. prospektive randomisierte kontrollierte pilotstudie," Handchir Mikrochir Plast Chir, vol. 40, pp. 66-73, Feb. 2008.

[21] J. Klein, S. Spencer, J. Allington, K. Minakata, E. Wolbrecht, R. Smith, J. Bobrow, and D. Reinkensmeyer, "Biomimetic orthosis for the neurorehabilitation of the elbow and shoulder (bones)," in BioRob, pp. 535-541, IEEE, 2008.

[22] M. Milot, S. Spencer, V. Chan, J. Allington, J. Klein, C. Chou, J. Bobrow, S. Cramer, and D. Reinkensmeyer, "A crossover pilot study evaluating the functional outcomes of two different types of robotic movement training in chronic stroke survivors using the arm exoskeleton bones," JNER, vol. 10, p. 112, Dec. 2013.

[23] T. Sakurada, T. Kawase, K. Takano, T. Komatsu, and K. Kansaku, "A bmi-based occupational therapy assist suit: asynchronous control by ssvep," Front Neurosci, vol. 7, 2013.

[24] M. Rahman, T. Ouimet, M. Saad, J. Kenne, and P. Archambault, 
"Development and control of a wearable robot for rehabilitation of elbow and shoulder joint movements," in IECON, pp. 1506-1511, IEEE, 2010

[25] M. Simkins, H. Kim, G. Abrams, N. Byl, and J. Rosen, "Robotic unilateral and bilateral upper-limb movement training for stroke survivors afflicted by chronic hemiparesis," in ICORR, pp. 1-6, IEEE, 2013

[26] Y. Ren, S. Kang, H. Park, Y. Wu, and L. Zhang, "Developing a multijoint upper limb exoskeleton robot for diagnosis, therapy, and outcome evaluation in neurorehabilitation," TNSRE, vol. 21, pp. 490-499, May 2013

[27] G. De Lee, W. Wang, K. Lee, S. Lin, L. Fu, J. Lai, W. Chen, and J. Luh, "Arm exoskeleton rehabilitation robot with assistive system for patient after stroke," in ICCAS, pp. 1943-1948, IEEE, 2012.

[28] S. Balasubramanian, R. Wei, M. Perez, B. Shepard, E. Koeneman, J. Koeneman, and J. He, "Rupert: An exoskeleton robot for assisting rehabilitation of arm functions," in Virtual Rehabilitation, 2008, pp. 163-167, IEEE, 2008.

[29] D. Caldwell, N. Tsagarakis, S. Kousidou, N. Costa, and I. Sarakoglou, "Soft exoskeleton for upper and lower body rehabilitation - desing, control and testing," INT J HUM ROBOT, vol. 4, no. 03, pp. 549-573, 2007.

[30] R. Gopura, K. Kiguchi, and Y. Li, "Sueful-7: A 7dof upper-limb exoskeleton robot with muscle-model-oriented emg-based control," in IROS, pp. 1126-1131, IEEE, 2009.

[31] S. Moubarak, M. T. Pham, T. Pajdla, and T. Redarce, "Design and modeling of an upper extremity exoskeleton," in World Congress on Medical Physics and Biomedical Engineering (O. Dssel and W. C. Schlegel, eds.), pp. 476-479, Springer Berlin Heidelberg, Jan. 2009.

[32] H. Lo and S. Xie, "An upper limb exoskeleton with an optimized 4 spherical wrist mechanism for the shoulder joint," in AIM, pp. 269-274, IEEE, 2014.

[33] P. Garrec, J. Friconneau, Y. Measson, and Y. Perrot, "Able an innovative transparent exoskeleton for the upper-limb," in IROS, pp. 1483-1488, IEEE, 2008

[34] V. Crocher, A. Sahbani, J. Robertson, A. Roby-Brami, and G. Morel, "Constraining upper limb synergies of hemiparetic patients using a robotic exoskeleton in the perspective of neuro-rehabilitation," TNSRE, vol. 20, no. 3, pp. 247-257, 2012.

[35] E. Pirondini, M. Coscia, S. Marcheschi, G. Roas, F. Salsedo, A. Frisoli, M. Bergamasco, and S. Micera, "Evaluation of a new exoskeleton for upper limb post-stroke neuro-rehabilitation: Preliminary results," in Replace, Repair, Restore, Relieve-Bridging Clinical and Engineering Solutions in Neurorehabilitation, pp. 637-645, Springer, 2014.

[36] M. Ergin and V. Patoglu, "Assiston-se: A self-aligning shoulder-elbow exoskeleton," in ICRA, pp. 2479-2485, IEEE, 2012.

[37] Y. Mao and S. Agrawal, "Design of a cable-driven arm exoskeleton (carex) for neural rehabilitation," TRO, vol. 28, pp. 922-931, Aug. 2012.

[38] J. Garrido, W. Yu, and A. Soria, "Modular design and modeling of an upper limb exoskeleton," in BioRob, pp. 508-513, IEEE, 2014.

[39] A. Frisoli, L. Borelli, A. Montagner, S. Marcheschi, C. Procopio, F. Salsedo, M. Bergamasco, M. Carboncini, M. Tolaini, and B. Rossi, "Arm rehabilitation with a robotic exoskeleleton in virtual reality," in ICORR, pp. 631-642, IEEE, 2007.

[40] A. Frisoli, C. Procopio, C. Chisari, I. Creatini, L. Bonfiglio, M. Bergamasco, B. Rossi, M. Carboncini, et al., "Positive effects of robotic exoskeleton training of upper limb reaching movements after stroke," JNER, vol. 9, no. 1, p. 36, 2012.

[41] A. Otten, A. Stienen, E. van Asseldonk, R. Aarts, and H. van der Kooij, "LIMPACT: a hydraulically powered self-aligning upper limb exoskeleton," 2013.

[42] S. Ball, I. Brown, and S. Scott, "Medarm: a rehabilitation robot with 5dof at the shoulder complex," in AIM, pp. 1-6, IEEE, 2007.

[43] C. Carignan, J. Tang, and S. Roderick, "Development of an exoskeleton haptic interface for virtual task training," in IROS, pp. 3697-3702, IEEE, 2009.

[44] G. Johnson, D. Carus, G. Parrini, S. Marchese, and R. Valeggi, "The design of a five-degree-of-freedom powered orthosis for the upper limb," Proc Inst Mech Eng H, vol. 215, pp. 275-284, Mar. 2001.

[45] E. Wolbrecht, D. Reinkensmeyer, and J. Bobrow, "Pneumatic control of robots for rehabilitation," Int. J. Robot. Res., vol. 29, pp. 23-38, May 2009.

[46] D. Reinkensmeyer, E. Wolbrecht, V. Chan, C. Chou, S. Cramer, and J. Bobrow, "Comparison of three-dimensional, assist-as-needed robotic $\mathrm{arm} / \mathrm{hand}$ movement training provided with pneu-wrex to conventional tabletop therapy after chronic stroke.," Am J Phys Med Rehabil, vol. 91, no. 11 Suppl 3, pp. S232-41, 2012.
[47] R. Vertechy, A. Frisoli, A. Dettori, M. Solazzi, and M. Bergamasco, "Development of a new exoskeleton for upper limb rehabilitation," in ICORR, pp. 188-193, IEEE, 2009.

[48] M. Gunasekara, R. Gopura, and S. Jayawardena, "6-rexos: Upper limb exoskeleton robot with improved phri," Int J Adv Robot Syst, pp. 12-47, 2015.

[49] A. Gupta and M. O'Malley, "Design of a haptic arm exoskeleton for training and rehabilitation," TMECH, vol. 11, pp. 280-289, June 2006

[50] M. Ding, J. Ueda, and T. Ogasawara, "Pinpointed muscle force control using a power-assisting device: system configuration and experiment," in BioRob, pp. 181-186, IEEE, 2008.

[51] Z. Song, S. Guo, M. Pang, S. Zhang, N. Xiao, B. Gao, and L. Shi, "Implementation of resistance training using an upper-limb exoskeleton rehabilitation device for elbow joint," J Med Biol Eng, vol. 34, no. 2, 2014.

[52] J. Martinez, P. Ng, S. Lu, M. Campagna, and O. Celik, "Design of wrist gimbal: A forearm and wrist exoskeleton for stroke rehabilitation," in ICORR, pp. 1-6, IEEE, 2013.

[53] J. Perry and J. Rosen, "Design of a 7 degree-of-freedom upper-limb powered exoskeleton," in BioRob, pp. 805-810, IEEE, 2006.

[54] R. Morales, F. Badesa, N. Garca-Aracil, J. Sabater, and C. Prez-Vidal, "Pneumatic robotic systems for upper limb rehabilitation," MED BIOL ENG COMPUT, vol. 49, pp. 1145-1156, Aug. 2011.

[55] A. Jackson, R. Holt, P. Culmer, S. Makower, M. Levesley, R. Richardson, J. Cozens, M. Williams, and B. Bhakta, "Dual robot system for upper limb rehabilitation after stroke: The design process," Proc. Inst. Mech. Eng. C J. Mech. Eng. Sci., vol. 221, pp. 845-857, July 2007.

[56] G. Rosati, P. Gallina, S. Masiero, and A. Rossi, "Design of a new 5 dof wire-based robot for rehabilitation," in ICORR, pp. 430-433, IEEE, 2005.

[57] A. Toth, G. Fazekas, G. Arz, M. Jurak, and M. Horvath, "Passive robotic movement therapy of the spastic hemiparetic arm with reharob: report of the first clinical test and the follow-up system improvement,' in ICORR, pp. 127-130, IEEE, 2005.

[58] R. Shadmehr and F. Mussa-Ivaldi, "Adaptive representation of dynamics during learning of a motor task," J Neurosci, vol. 14, no. 5, pp. 3208-3224, 1994.

[59] Y. Wei, P. Bajaj, R. Scheidt, and J. Patton, "Visual error augmentation for enhancing motor learning and rehabilitative relearning," in ICORR 2005, pp. 505-510, IEEE, 2005.

[60] P. Staubli, T. Nef, V. Klamroth-Marganska, and R. Riener, "Jner," JNER, vol. 6, p. 46, 2009.

[61] T. Nef, M. Mihelj, and R. Riener, "Armin: a robot for patientcooperative arm therapy," MED BIOL ENG COMPUT, vol. 45, pp. 887-900, Sept. 2007.

[62] S. Moubarak, M. Pham, R. Moreau, and T. Redarce, "Gravity compensation of an upper extremity exoskeleton," in EMBC, pp. 4489-4493, IEEE, 2010.

[63] Z. Li, C. Su, G. Li, and H. Su, "Fuzzy approximation-based adaptive backstepping control of an exoskeleton for human upper limbs,"

[64] S. Kousidou, N. G. Tsagarakis, C. Smith, and D. G. Caldwell, "Taskorientated biofeedback system for the rehabilitation of the upper limb," in ICORR, pp. 376-384, IEEE, 2007.

[65] C. Loconsole, S. Dettori, A. Frisoli, C. Avizzano, and M. Bergamasco, "An emg-based approach for on-line predicted torque control in roboticassisted rehabilitation," in HAPTICS, pp. 181-186, IEEE, 2014.

[66] M. Rahman, C. Ochoa-Luna, M. Saad, and P. Archambault, "Emg based control of a robotic exoskeleton for shoulder and elbow motion assist," J Aut Con Eng, vol. 3, no. 4, 2015.

[67] E. Wolbrecht, V. Chan, D. Reinkensmeyer, and J. Bobrow, "Optimizing compliant, model-based robotic assistance to promote neurorehabilitation," TNSRE, vol. 16, pp. 286-297, June 2008.

[68] R. Wei, S. Balasubramanian, L. Xu, and J. He, "Adaptive iterative learning control design for rupert iv," in BioRob, pp. 647-652, IEEE, 2008.

[69] A. Frisoli, C. Loconsole, R. Bartalucci, and M. Bergamasco, "A new bounded jerk on-line trajectory planning for mimicking human movements in robot-aided neurorehabilitation," Rob Auton Syst, vol. 61 , no. 4, pp. 404-415, 2013.

[70] E. Guigon, P. Baraduc, and M. Desmurget, "Computational motor control: redundancy and invariance," J. Neurophysiol., vol. 97, no. 1, pp. 331-347, 2007.

[71] S. Guo, W. Zhang, W. Wei, J. Guo, Y. Ji, and Y. Wang, "A kinematic model of an upper limb rehabilitation robot system," in ICMA, pp. 968 973, IEEE, 2013. 
[72] A. Frisoli, C. Loconsole, D. Leonardis, F. Banno, M. Barsotti, C. Chisari, and M. Bergamasco, "A new gaze-bci-driven control of an upper limb exoskeleton for rehabilitation in real-world tasks," IEEE TSMC, vol. 42, no. 6, pp. 1169-1179, 2012.

[73] M. Barsotti, D. Leonardis, C. Loconsole, M. Solazzi, E. Sotgiu, C. Procopio, C. Chisari, M. Bergamasco, and A. Frisoli, "A full upper limb robotic exoskeleton for reaching and grasping rehabilitation triggered by mi-bci," in ICORR, pp. 49-54, IEEE, 2015.

[74] D. Brauchle, M. Vukelić, R. Bauer, and A. Gharabaghi, "Brain-state dependent robotic reaching movement with a multi-joint arm exoskeleton: combining brain-machine interfacing and robotic rehabilitation," Front. Hum. Neurosci., vol. 9, p. 564, 2015.

[75] D. Lynch, M. Ferraro, J. Krol, C. M. Trudell, P. Christos, and B. T. Volpe, "Continuous passive motion improves shoulder joint integrity following stroke," $C R E$, vol. 19, no. 6, pp. 594-599, 2005.

[76] N. Hogan, H. Krebs, B. Rohrer, J. Palazzolo, L. Dipietro, S. Fasoli, J. Stein, R. Hughes, W. R. Frontera, D. Lynch, et al., "Motions or muscles? some behavioral factors underlying robotic assistance of motor recovery," JRRD, vol. 43, no. 5, p. 605, 2006.

[77] J. Patton and F. Mussa-Ivaldi, "Robot-assisted adaptive training: custom force fields for teaching movement patterns," TBME, vol. 51, no. 4, pp. 636-646, 2004.

[78] N. Hogan and H. Krebs, "Interactive robots for neuro-rehabilitation," Restor. Neurol. Neurosci., vol. 22, no. 3, pp. 349-358, 2004

[79] N. Hogan, "Impedance control: An approach to manipulation," in ACC, 1984, pp. 304-313, 1984.

[80] P. Culmer, A. Jackson, S. Makower, R. Richardson, J. Cozens, M. Levesley, and B. Bhakta, "A control strategy for upper limb robotic rehabilitation with a dual robot system," TMECH, vol. 15, pp. 575-585, Aug. 2010.

[81] B. Tsai, W. Wang, L. Hsu, L. Fu, and J. Lai, "An articulated rehabilitation robot for upper limb physiotherapy and training," in IROS, pp. 1470-1475, Oct. 2010.

[82] A. Frisoli, F. Salsedo, M. Bergamasco, B. Rossi, and M. Carboncini, "A force-feedback exoskeleton for upper-limb rehabilitation in virtual reality," Appl Bionics Biomech, vol. 6, no. 2, pp. 115-126, 2009.

[83] W. Yu, J. Rosen, and X. Li, "Pid admittance control for an upper limb exoskeleton," in ACC, pp. 1124-1129, IEEE, 2011.

[84] L. Miller and J. Rosen, "Comparison of multi-sensor admittance control in joint space and task space for a seven degree of freedom upper limb exoskeleton," in BioRob, pp. 70-75, IEEE, 2010.

[85] H. Kim, L. Miller, I. Fedulow, M. Simkins, G. Abrams, N. Byl, and J. Rosen, "Kinematic data analysis for post-stroke patients following bilateral versus unilateral rehabilitation with an upper limb wearable robotic system," TNSRE, vol. 21, no. 2, pp. 153-164, 2013.

[86] H. Kim, Z. Li, D. Milutinovic, and J. Rosen, "Resolving the redundancy of a seven dof wearable robotic system based on kinematic and dynamic constraint," in ICRA, pp. 305-310, IEEE, 2012.

[87] M. Ding, K. Hirasawa, Y. Kurita, H. Takemura, J. Takamatsu, H. Mizoguchi, and T. Ogasawara, "Pinpointed muscle force control in consideration of human motion and external force," in ROBIO, pp. 739 744, IEEE, 2010.

[88] H. Zhang, H. Austin, S. Buchanan, R. Herman, J. Koeneman, and J. He, "Feasibility study of robot-assisted stroke rehabilitation at home using rupert," in $C M E$, pp. 604-609, IEEE, 2011.

[89] T. Proietti, N. Jarrasse, A. Roby-Brami, and G. Morel, "Adaptive control of a robotic exoskeleton for neurorehabilitation," in NER, 2015.

[90] A. Pehlivan, D. Losey, and M. OMalley, "Minimal assist-as-needed controller for upper limb robotic rehabilitation," Robotics, IEEE Transactions on, vol. 32, pp. 113-124, Feb 2016.

[91] R. Pérez-Rodríguez, C. Rodríguez, Ú. Costa, C. Cáceres, J. M. Tormos, J. Medina, and E. J. Gómez, "Anticipatory assistance-as-needed control algorithm for a multijoint upper limb robotic orthosis in physical neurorehabilitation," Expert Systems with Applications, vol. 41, no. 8, pp. 3922-3934, 2014.

[92] Y. Mao, X. Jin, G. Dutta, J. Scholz, and S. Agrawal, "Human movement training with a cable driven arm exoskeleton (carex)," TNSRE, vol. 23, no. 1, pp. 84-92, 2014.

[93] Y. Mao and S. Agrawal, "A cable driven upper arm exoskeleton for upper extremity rehabilitation," in ICRA, pp. 4163-4168, IEEE, 2011.

[94] N. Bernstein, The coordination and regulation of movement. 1967.

[95] E. Brokaw, T. Murray, T. Nef, and P. Lum, "Retraining of interjoint arm coordination after stroke using robot-assisted time-independent functional training," JRRD, vol. 48, no. 4, pp. 299-316, 2011.

[96] E. Brokaw, D. Nichols, R. Holley, and P. Lum, "Robotic therapy provides a stimulus for upper limb motor recovery after stroke that is complementary to and distinct from conventional therapy," $N N R$, p. 1545968313510974, 2013.

[97] M. Guidali, M. Schmiedeskamp, V. Klamroth, and R. Riener, "Assessment and training of synergies with an arm rehabilitation robot," in ICORR, pp. 772-776, IEEE, 2009.

[98] N. Jarrasse, M. Tagliabue, J. Robertson, A. Maiza, V. Crocher, A. RobyBrami, and G. Morel, "A methodology to quantify alterations in human upper limb movement during co-manipulation with an exoskeleton," TNSRE, vol. 18, no. 4, pp. 389-397, 2010.

[99] J. Fong, V. Crocher, D. Oetomo, and Y. Tan, "An investigation into the reliability of upper-limb robotic exoskeleton measurements for clinical evaluation in neurorehabilitation," in NER, 2015.

[100] N. Nordin, S. Xie, and B. Wünsche, "Assessment of movement quality in robot-assisted upper limb rehabilitation after stroke: a review," JNER, vol. 11, no. 1, p. 137, 2014.

[101] R. Vertechy, A. Frisoli, M. Solazzi, D. Pellegrinetti, and M. Bergamasco, "An interaction-torque controller for robotic exoskeletons with flexible joints: Preliminary experimental results," in IROS, pp. 335-340, IEEE, 2012

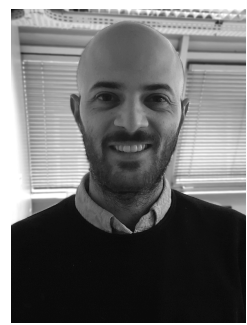

Tommaso Proietti received his B.Sc. and M.Sc. from La Sapienza University of Rome, Italy, in 2010 and 2013 respectively. He was Visiting Pre-Doctoral Fellow at the Neuroscience and Robotics Laboratory at the Northwestern University, IL, USA, from October 2012 to October 2013, working on dynamic nonprehensile manipulation. He is currently $\mathrm{Ph} . \mathrm{D}$. candidate in Robotics Engineering at the University Pierre et Marie Curie in Paris, France. His Ph.D deals with control strategies for robotic exoskeletons, in the field of stroke neurorehabilitation and human motor control learning. His research interests include physical Human-Robot Interaction, rehabilitation robotics, biomimetic systems, and haptics.

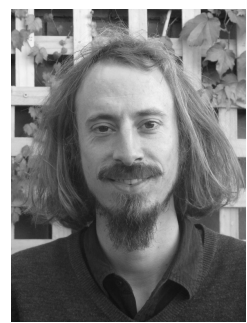

Vincent Crocher received the Diploma of Engineer in Computer Science and Electronics from Polytech Paris-UPMC in 2008 and obtained his PhD in Robotics in 2012 from the University Pierre et Marie Curie (Paris, France). His PhD research project was on the development and evaluation of robotics exoskeleton control for neuro-rehabilitation. He has been working at The Hand Rehabilitation Lab in Milwaukee (Wisconsin, USA) and is now continuing his research on robotics and virtual reality systems applied to neuro-rehabilitation as a Postdoctoral Research Fellow at The University of Melbourne.

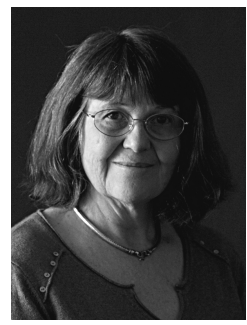

Agnès Roby-Brami received the M.D. degree from the University Paris Descartes, in 1982, and M.Sc. and $\mathrm{Ph} . \mathrm{D}$. degrees and University Habilitation in Neurosciences from Pierre et Marie Curie University, Paris, France, in 1982, 1991, and 2001, respectively. She is a physician who performed her residency in Paris Hospitals during 1977-1982. She was appointed as a Research Associate at INSERM in 1983 and promoted to Research Director in 2005 Her first research interest was in the field of clinical neurophysiology after which she focused on upper limb motor control, disability, and recovery in cerebral and spinal injured patients. She is the author or coauthor of 92 peer-reviewed academic publications, and 44 contributions to edited books. She is also actively involved in several Academic Networks concerning research on disability. 


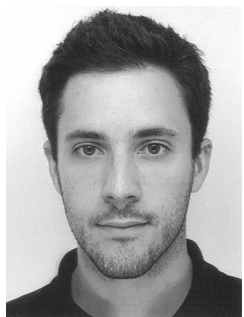

Nathanaël Jarrassé received an M.Sc. in Industrial Systems Engineering from ENSAM, and an M.Sc. and a Ph.D. in Robotics from UPMC, Paris. He has been a postdoctoral Research Associate at the HRG, Department of Bioengineering of Imperial College London, and is now a Tenured Researcher for the National Center for Scientific Research (CNRS) at ISIR. His research focuses on physical human-robot interaction for medical applications, and aims at developing robotic interactive systems (prostheses, exoskeletons, instrumented objects) to study and characterize the human sensorimotor system, and to improve assistance and rehabilitation of individuals affected by a motor skills loss. 